\title{
Micro total analysis systems in biopharmaceutical process development
}

\author{
"To fulfill the promise of micro total analysis systems in the biopharmaceutical industry, it is important to bridge academic \\ advancements with industry needs through collaborations of scientists from academia, instrument vendors and \\ industry laboratories."
}

Micro total analysis systems ( $\mu$ TAS), better known as 'lab-on-a-chip' devices, are miniaturized devices with integrated functionalities including sample loading, sample treatment, separation and detection. A wide variety of applications have been reported using $\mu$ TAS, from DNA sequencing to protein purity analysis, from cell cultivation to immunoassay, from diagnosis to drug screening and development. Some recent reviews are available on $\mu$ TAS $[1]$ and their applications in drug discovery [2]. This article will focus on the utility of $\mu$ TAS in process development in the biopharmaceutical industry and under the broadened scope of 'bioanalysis', for quantification and characterization of protein therapeutics.

Although various formats and capabilities of $\mu$ TAS have been demonstrated in scientific literature and explored in academic settings, only a limited number of platforms are commercially available for implementation in industry laboratories [3]. When compared with their macroscale counterparts, HPLC or CE for example, $\mu$ TAS have a few advantages that make them attractive technologies to the biopharmaceutical industry: economic to operate with low solvent/reagent consumption; environmentally friendly with less waste generated; and, more importantly, capable of high-throughput analysis with low sample consumption and fast separation.

\section{Nice to have or a necessity?}

There are two common concerns when adopting new technologies in the pharmaceutical industry. One is the risk of investing in a rising technology that has not been tested over an extended period of time. The other is associated with the potential questioning from regulatory agencies regarding correlations with other well-established techniques. Those boundaries, however, seem to be dissolving, driven by efforts from both ends. On one hand, to succeed in today's competitive environment, biopharmaceutical companies need to improve their efficiency more than ever by adopting enabling technologies. On the other hand, regulatory agencies are encouraging innovations to increase the overall understanding of the process and the product. This is evidenced by some recent initiatives aimed at ensuring the quality of therapeutic drugs, such as the US FDA's Pharmaceutical cGMPs for the 21st Century [101] and Quality by Design [102]. There is a clear synergy to utilize innovative technologies that can provide patients with high-quality drugs at lower costs based on sound scientific evidence and well-calculated risks.

\section{"Although various formats and capabilities of} $\mu$ TAS have been demonstrated in scientific literature ... only a limited number of platforms are commercially available for implementation in industry laboratories."

To become the front runner and maintain the competitive edge in the industry, biopharmaceutical companies are striving to improve the speed to market for new drugs, lower the cost and meet ever-increasing quality standards at the same time. Researchers in biopharmaceutical companies have to face the consequent challenges of moving more drug candidates to the later stages of development with shortened timelines, gaining greater understanding of the process with fewer resources or, in general, doing more with less. At least part of the answer to such challenges lies in high-throughput discovery and development. High-throughput technology is no longer a future perspective that is only nice to have; it is becoming more and

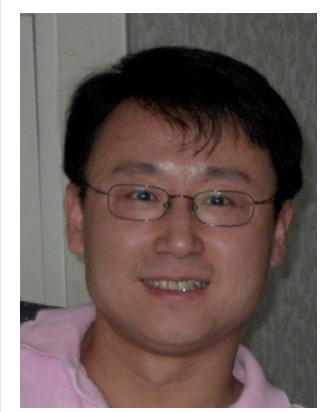

Xiaoyu Chen

Process Sciences and

Production, Novartis

Biologics, 200 Technology

Square, Cambridge,

MA 02139, USA

Tel.: + 617 87| 7152

E-mail: xiaoyu-I.chen@

novartis.com
FUTURE
SCIENCE 
more important in daily operations of process development, whether it is for the development of upstream or downstream processing, clone selection or formulation screening. With the advancement in microbioreactors [4,5] and highthroughput purification platforms [6,7], hundreds and thousands of samples can be generated in a short period of time. A great number of vector constructs, strains or clones can be evaluated in a massively parallel fashion and a wide range of resins and purification conditions can be explored within a single experiment. The outcome of those experiments, however, relies on high-throughput analytical techniques as the capacity of a typical analytical laboratory can be easily exceeded. In fact, more and more highthroughput analytical laboratories are being established in the industry, aiming at improving the efficiency by adopting enabling technologies to fulfill the ever-increasing analytical demands.

\section{"Microfluidic-based analytical techniques using commercial platforms are available for analysis of therapeutic proteins, including binding assays on microfluidic CDs, LC and electrophoresis on microfluidic chips."}

High-throughput analysis is also a valuable tool for the pharmaceutical industry during the current transitioning from the traditional era of quality by testing into the new era of quality by design (QbD). Pharmaceutical QbD is a science- and risk-based systematic approach to ensure the quality of drug product. The fundamental principle of $\mathrm{QbD}$ is that quality cannot be tested into products (after being manufactured) but should be built-in by proper design of the complete manufacturing process. Upon implementation, the quality of a protein therapeutic will be assured by controlling the process parameters during manufacturing (e.g., fermentation $\mathrm{pH}$ and purification column temperature) rather than by the analytical tests performed on the final product. The key factor in $\mathrm{QbD}$ is the detailed understanding of the process, particularly the relationship between two spaces: the quality space of the drug product defined by its critical quality attributes (CQAs) and the design space of the process defined by its critical process parameters (CPPs). The flexibility allowed for a given process design space can only be granted with detailed understanding of the quality profile within. The linkage between CQAs and CPPs needs to be established in early development. Whereas analytical testing may only become confirmatory for material release resulting in reduced end-product testing, considerable investment in analytical activities has to be made up-front in the development to understand the impact of the process on product quality. Comprehensive process understanding is not a trivial task in the biopharmaceutical industry considering the natural variations in biological process and lot-to-lot variability of raw materials. Large-scale design of experiments must be performed exploring the limits of individual process parameters as well as their combinations. A great number of samples can be quickly generated for quality assessment. Assuming an average testing time of $30 \mathrm{~min}$ per sample, 1000 samples would take 21 days of instrument time using conventional HPLC or CE methods. The instrument time can be shortened to 1 working day if each test can be performed within $30 \mathrm{~s}$. High-throughput analysis using $\mu$ TAS has the potential to fully support the QbD initiative.

\section{Example applications: microchip CE-sodium dodecyl sulfate}

A few microfluidic-based analytical techniques using commercial platforms are available for analysis of therapeutic proteins, including binding assays on microfluidic CDs, LC and electrophoresis on microfluidic chips. Microchip CEsodium dodecyl sulfate (SDS) is one of the more popular high-throughput analytical techniques used in today's biotechnology laboratories and will be discussed as an example. Besides protein quantification, microchip CE-SDS also provides purity assessment including levels of fragmentation and covalent aggregation, assembly of the product, glycan occupancy and, recently, glycan class and total dimer levels $[8,9]$.

In microchip CE-SDS analysis, low micrograms or submicrograms of proteins are sufficient for sample preparation. Each sample separation takes approximately $40 \mathrm{~s}$ and the total instrument time per sample is less than $1 \mathrm{~min}$. The high separation efficiency of such microchip CE-SDS is a direct benefit of the small dimensions of the microfluidic channels: superior heat transfer due to large surface-to-volume ratio, and small and precisely controlled sample plug length. In addition, sample preparation can be performed in 96- or 384-well plates using multichannel pipettes or liquid handlers and a sipper chip design allows automatic sample loading. Combined with automatic data processing, hundreds of samples can be analyzed in a few 
hours using a microchip CE-SDS instrument with minimal analyst intervention, in contrast to a few days on a conventional CE-SDS instrument. It is also shown that microchip CE-SDS provides sensitivity and resolution comparable with that of conventional CE-SDS analysis [8]. The high speed of the analysis with adequate performances makes this technique well suited for high-throughput quantitation and purity assessments.

In addition to titer estimation, microchip CE-SDS can also provide purity profiles of crude cell culture or fermentation samples. Addressing quality attributes in early process development is becoming more and more critical as any late changes of the process are inevitably costly. During early strain or clone screening or upstream process development, the improvement in production should be carefully balanced with the quality of the material. Removing productrelated impurities by downstream purifications could lead to longer process development time, significant losses in process yield and sometimes may not even be feasible. It is, however, still a common practice to rank different strains, clones or cell culture/fermentation conditions based on the expression level only and quality assessment is only made for the final few candidates that are purified and then analyzed by conventional analytical techniques. Two factors could contribute to improving the throughput of the screening processes: speed of the analytical testing itself and the capability of analyzing crude samples either directly or with simple pretreatment that can be performed in micro-well plates. Some recent examples include glycan typing and total dimer screening of antibody cell culture samples by microchip electrophoresis [8,9]. With such high-throughput analytics, especially the capability of performing purity measurement on crude samples, it is now possible to incorporate both productivity and purity factors into the process of candidate screening and upstream development, which in turn reduces the risks associated with later process changes.

\section{Future perspective}

Although microchip electrophoresis is becoming more routinely used in biotechnology laboratories to obtain simultaneous titer and purity information for both upstream and downstream samples, there are still areas of further improvement on commercial platforms. Higher sensitivity is needed to quantify low-expressing proteins produced in deep-well plates or microbioreactors during early screening process. Extended dynamic range is desired to identify and quantify minor fragments in the presence of main peak species early in formulation screening. Another area of development is to expand the scope of the product quality attributes that can be addressed in high-throughput fashion, ideally for samples in crude cell culture or fermentation broth. For example, it is desirable to also include the distribution of charged variants, the glycosylation pattern, the level of total aggregation and disulfide connectivity in the purity space during screening processes. Interfacing with MS is another attractive direction to extend the capabilities of $\mu$ TAS. Coupling or preferably converting the outlet of the microfluidic separation channel to a nanospray tip will allow online UV/fluorescence and MS detection for simultaneous purity assessment and detailed characterization. HPLC chips coupled to MS are now commercially available. The ability to interface MS with other modes of microchip separation such as electrophoresis, although successfully demonstrated in academic laboratories [10], remains to be developed for commercial instruments.

\section{"Another area of development is to expand the scope of the product quality attributes that can be addressed in high-throughput fashion, ideally for samples in crude cell culture or fermentation broth."}

Whereas $\mu$ TAS have had great success in DNA analysis by incorporating PCR amplification, separation and detection all on a single device, the integration of different functionalities for protein analysis is much less mature. Due to the diversity in size, abundance and post-translational modifications, protein sample treatment and separation are far more complicated. Such integration, however, is highly desirable to take full advantage of the high detection sensitivity and to improve the speed of overall analysis. It is not uncommon that the amount of sample required for sample preparation (often in microliters) is orders of magnitude higher than the actual amount of sample producing the signal (often in picoliters) in a microfluidic chip. The sensitivity of the detection can only be fully realized when sample preparation steps are integrated onto the miniaturized device to improve the overall sensitivity. On-chip sample treatment can also greatly reduce the total analysis time. For example, it has been shown in some proof-of-concept studies 
that enzymatic digestion in the microreactor of a microfluidic device takes only seconds instead of hours [11,12]. The digests can then be enriched, separated and detected on the same chip, a great improvement in both overall sensitivity and total analysis time. A broader range of sample preparations integrated with separation and detection on a single commercial chip based on specific needs can be anticipated in the near future.

In addition to high-throughput screening during process development, $\mu$ TAS may also be suitable for GMP-compliant quality control (QC). Just like many other analytical techniques, software in compliance with regulations and consumables with appropriate quality specifications are all prerequisites for the implementation of $\mu$ TAS in quality laboratories. Once an assay is shown to be robust and qualified in the analytical development laboratory, it may also be validated in a QC unit according to the procedures established for conventional methods.

Another stage for $\mu$ TAS is real-time analysis during production. The implementation of $\mathrm{QbD}$ relies on enabling process analytical technology (PAT). Whereas PAT is a broad concept, timely measurement of CQAs of in-process materials is an important element. With the detailed understanding of the process, CPPs can be adjusted 'on-the-fly' with the feedback of real-time analysis by PAT. In addition to the spectroscopic and chromatographic methods, $\mu$ TAS, with automatic sample loading, sample treatment, detection and readouts, also has the potential to be used on-line or at-line during manufacturing to provide real-time feedback on the process performance.

In conclusion, $\mu$ TAS have become invaluable tools in biopharmaceutical development, driven by both scientific and regulatory perspectives. To fulfill the promises of $\mu$ TAS in the biopharmaceutical industry, it is important to bridge academic advancements with industry needs through collaborations of scientists from academia, instrument vendors and industry laboratories. The utility of $\mu$ TAS will surely continue to grow with higher sensitivity and dynamic range, broader coverage of quality attributes and better integration of different functionalities.

\section{Financial \& competing interests disclosure}

The author has no relevant affliations or financial involvement with any organization or entity with a financial interest in or financial conflict with the subject matter or materials discussed in the manuscript. This includes employment, consultancies, honoraria, stock ownership or options, expert testimony, grants or patents received or pending, or royalties.

No writing assistance was utilized in the production of this manuscript.

\section{Bibliography}

1 West J, Becker M, Tombrink S, Manz A. Micro total analysis systems: latest achievements. Anal. Chem. 80, 4403-4419 (2008).

2 Dittrich PS, Manz A. Lab-on-a-chip: microfluidics in drug discovery. Nat. Rev. Drug Discov. 5, 210-218 (2006).

3 Clayton J. Go with the microflow. Nat. Meth. 2, 621-627 (2005).

4 Schreyer HB, Miller SE, Rodgers S. Application note: high-throughput process development. Gen. Eng. News 27(17), (2007).

5 Gefen O, Balaban NQ. The Moore's law of microbiology - towards bacterial culture miniaturization with the micro-Petri chip. Trends Biotechnol. 26, 345-347 (2007).

6 Smith C. Striving for purity: advances in protein purification. Nat. Meth. 2, 71-77 (2005).
7 Coffman JL, Kramarczyk, JF, Kelley BD. High-throughput screening of chromatographic separations: I. Method development and column modeling. Biotechnol. Bioeng. 100, 605-618 (2008).

8 Chen X, Tang K, Lee M, Flynn G. Microchip assays for screening monoclonal antibody product quality. Electrophoresis 29, 4993-5002 (2008).

9 Chen X, Flynn G. A high throughput dimer screening assay for monoclonal antibodies using chemical cross-linking and microchip electrophoresis. J. Chromatogr. B. 877, 3012-3018 (2009)

10 Gaspar A, Englmann M, Fekete A, Harir M, Schmitt-Kopplin P. Trends in CE-MS 2005-2006. Electrophoresis 29, 66-79 (2008).

11 Bynum MA, Yin H, Felts K, Lee YM, Monell CR, Killeen K. An integrated microfluidic LC/MS chip for rapid on-line deglycosylation and characterization of $N$-glycans from recombinant IgG antibodies. Presented at: The 57th ASMS Conference on Mass Spectrometry. 31 May-4 June, PA, USA, 2009.

12 Liuni P, Rob T, Wilson DJ. A chip-based proteolytic reactor for ESI-MS analysis. Presented at: The 57th ASMS Conference on Mass Spectrometry. 31 May-4 June, PA, USA, 2009.

\section{Websites}

101 US FDA. Pharmaceutical cGMPs for the 21st Century - a risk-based approach, final report (2004) www.fda.gov/downloads/drugs/ developmentapprovalprocess/manufacturing/ questionsandanswersoncurrent goodmanufacturingpracticescgmpfordrugs/ ucm 176374.pdf

102 US FDA. Guidance for industry: Q8(R1) Pharmaceutical Development (2009) www.fda.gov/downloads/drugs/ guidancecomplianceregulatoryinformation/ guidances/ucm073507.pdf 